\title{
Preliminary Risk Assessment of Trace Metal Pollution in Surface Water from Yangtze River in Nanjing Section, China
}

\author{
B. Wu $\cdot$ D. Y. Zhao $\cdot$ H. Y. Jia $\cdot$ \\ Y. Zhang $\cdot$ X. X. Zhang $\cdot$ S. P. Cheng
}

Published online: 26 February 2009

(C) Springer Science+Business Media, LLC 2009

\section{Erratum to: Bull Environ Contam Toxicol DOI 10.1007/s00128-008-9497-3}

There are errors in Table 1 and in Fig. 2. The corrected Table 1 and Fig. 2 appear below.

Table 1 Summary statistics of the analytical results of trace metals in water samples from Yangtze River $(\mu \mathrm{g} / \mathrm{L})$

\begin{tabular}{lccrl}
\hline Elements & Limit of detection & Range & Mean & SD \\
\hline Arsenic (As) & 3.6 & $7.6-20.8$ & 13.2 & 4.38 \\
Boron (B) & 5 & $28.4-55.6$ & 37.2 & 7.9 \\
Barium (Ba) & 3 & $32.9-41.3$ & 37.4 & 2.5 \\
Beryllium (Be) & 0.2 & $0.4-1.4$ & 0.5 & 0.3 \\
Cadmium (Cd) & 1 & $3.2-6.4$ & 4.7 & 0.91 \\
Chromium (Cr) & 4 & $17.2-24.3$ & 20.9 & 2.1 \\
Copper (Cu) & 4 & $8.6-12.3$ & 10.7 & 1.2 \\
Iron (Fe) & 10 & $174.5-350.5$ & 239.8 & 56.1 \\
Lead (Pb) & 30 & $44-734$ & 55.1 & 8.6 \\
Lithium (Li) & 5 & $13.1-15.6$ & 14.1 & 0.8 \\
Manganese (Mn) & 1 & $4.3-8.8$ & 5.4 & 1.6 \\
Molybdenum (Mo) & 5 & $6-19.8$ & 11.7 & 3.9 \\
\hline
\end{tabular}

The online version of the original article can be found under doi:10.1007/s00128-008-9497-3.

B. Wu $\cdot$ D. Y. Zhao $\cdot$ H. Y. Jia $\cdot$ Y. Zhang

X. X. Zhang $\cdot$ S. P. Cheng $(\square)$

State Key Laboratory of Pollution Control and Resource Reuse,

School of the Environment, Nanjing University, 210093

Nanjing, People's Republic of China

e-mail: chengsp@nju.edu.cn

B. Wu

e-mail: pandanju@gmail.com
Table 1 continued

\begin{tabular}{lccrc}
\hline Elements & Limit of detection & Range & Mean & SD \\
\hline Nickel (Ni) & 5 & $5.6-24.3$ & 13.4 & 4.9 \\
Antimony (Sb) & 20 & $49.5-86.9$ & 65.3 & 11.6 \\
Selenium (Se) & 5 & $5.6-170.7$ & 114.3 & 46.3 \\
Tin (Sn) & 10 & $58.5-117.8$ & 91.1 & 17.7 \\
Strontium (Sr) & 1 & $191.1-215.8$ & 210.1 & 6.6 \\
Vanadium (V) & 5 & $9.7-12$ & 10.5 & 0.7 \\
Zinc (Zn) & 5 & $7.6-11.6$ & 9.4 & 1.2 \\
Total metals & & $825.1-950.4$ & 929.29 & 37.67 \\
\hline
\end{tabular}

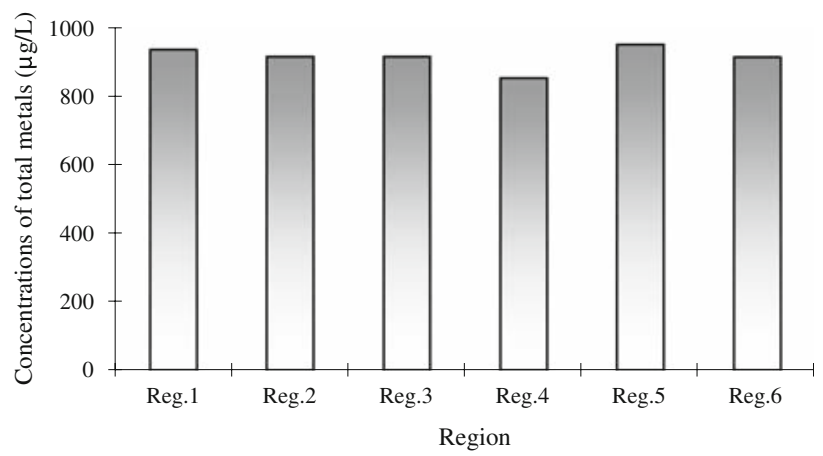

Fig. 2 Total concentrations of nineteen metals in different regions $(\lg / \mathrm{L})$ 